Medicine Updates Faculty of medicine October 2021, volume 7, issue 7 https://muj.journals.ekb.egdean@med.psu.edu.eg vice_dean_postgraduate@med.psu.edu.eg

DOI: 10.21608/MUJ.2021.96037.1065

ISSN : 2682-2741

\title{
" How to use quality tools to improve inpatient healthcare services in hospitals? "
}

\section{$\underline{\text { Authors }}$}

Aya Gamal Soliman ${ }^{-1}$ 1; Menna allah Mohamed²; Amr Abd Elaziz Alsaid3; Nancy Gamal Ahmed"; Gehad Nasser Ali ${ }^{4}$; Aya Ebrahim Kamel $^{4}$; Aya Salah Abd elraouf ${ }^{4}$; Rehab Hassan Hassan ${ }^{4}$; Heba Elsayed $^{5}$; Nourhan Abas ${ }^{6}$ -

\author{
${ }^{1}$ Bachelor of nursing Mansoura University, El Mansoura, Egypt. \\ ${ }^{2}$ Bachelor of pharmacy Sinai University, El Arish, Egypt. \\ ${ }^{3}$ Bachelor of Pharmacy Suez Canal University, Ismailia, Egypt. \\ ${ }^{4}$ Bachelor degree of Nursing from Port-said University, Egypt. \\ ${ }^{5}$ Port Said University, port said, Egypt. \\ ${ }^{6}$ Bachelor degree of pharmacy Suez canal, port said ,Egypt
}

\begin{abstract}
:
Healthcare institution is one of the institutions that mostly need quality in service. By improving the quality of service to it patients, a hospital would likely gain more trust from the patients, and moreover, would also get more patients' satisfaction. To find out the level of quality that has been done in hospitals, We create a study for the using of quality techniques and tools used to manage all of operations of the healthcare institutions is needed. The purpose of this study is to
\end{abstract}


map Quality Techniques and tools use in healthcare institutions. We assume that if the hospitals are using the quality tools and techniques, then the effectiveness and efficiency of the hospital will be improved hospitals .

The most used tools and techniques in the hospitals are fishbone, brainstorming and process mapping. Some reasons why the quality tools and techniques are not commonly used are because they don't have time to do the processes needed, they don't know how to use the quality tools and techniques, and measurements are not possible to do. Comparing use of these quality tools and techniques in hospitals in other countries, we can conclude that actually the quality tools and techniques use can give huge positive impacts to the hospitals. There are still a lot of room for improvement in hospitals.

\section{Key words}

(patient satisfaction, health Centre, fishbone, quality control, effectiveness and efficiency, communication, risk management ). 


\section{Introduction}

Quality control has become one of most important requirements recent years, and it has been a priority for many countries and World Health Organization's always ensure implementation of effective plans for quality of patient care within each healthcare system in regular way, most of countries are focus on implementation of quality management to deliver the best possible care delivered to patients, for achieving the positive clinical results, avoid clinical complications, and ensure efficient and effective use of resources available .

Also, quality tools have a great rule in improving the efficiency and effectiveness of the hospital industry. The goal is not only addition to reducing medical errors in health care process, as well as other positive effects that are important for health care quality. They include all the steps of the healthcare system itself, such as accuracy or speed of time, cost, quality of service or care, efficiency of physicians, and patient satisfaction (Fang and Manuel, 2007).

the quality of care improvement was useful for lowering waste times and the hospital errors, and maintaining patient satisfaction. Lowering cost was helped in lower the cost of care to the hospital, the patients.

Developing a hypothesis was that the hospitals in the quality group who would use the methodologies and tools more useful than those who not use quality group, and also the quality group would have a high positive level of patient and financial outcomes compared with who not use quality group.

Improvement of Quality starts by quality hospitals was different than those who not use quality group for both patient satisfaction and patient outcomes.

That make a conclusion that improving of quality starts by quality hospitals that lead to increasing patient satisfaction and patient outcomes, and had a greater positive effect on the financial measures when compared with who not use quality group hospitals. 


\section{The aim of the work}

The improvement of Quality study aimed at make positive outcomes in health care process to effecting desirable outcomes by use the Plan Do Study Act to achieve the goals of the project to Improve patients satisfaction,saving time, Patient Safety in inpatient .

1-used as an indicator of efficiency by reducing patient length of stay (LOS) by care coordination, transition and discharge planning

Delays in the discharge for patients can lead to increase in length of stay of patients, and increase the patient waiting list time, bed occupancy and thereby rate that increasing the cost for patient and hospital. The discharging process of patients is complicated, require coordination of different groups, including physicians, nurses, auxiliary services staff, patients' families, and sometimes the finance or billing departments. This study provides a number of measures that are helpful for hospital to control the improvement of length of stay.

2-improve the bed turnover.

3-The Morbidity \& the Mortality (M\&M) are important components for clinical setting. It has the importance to improve patient outcomes, the quality of care, performances for patient safety and they should concern and focus on the education of clinical staff.

4-impact of applying Clinical protocols in hospitals which help health care practitioners to apply right diagnostic treatment and right health care services to patients.

5- The involvement of pharmacists by performing clinical therapy management help to reduce medication related adverse effects in health care setting.

\section{Methodology}


THE Research team used the following quality tools to improve inpatient healthcare. The study of healthcare criteria needs identify of six sigma, the quality improvement focus on the hospitalized patients, the descriptions of project improvement and the results reported, all depend on the quality data reported.

Tool is Fishbone diagrams (Cause-and-effect diagrams) popularized in the 1960s by Kaoru Ishikawa, the pioneer of quality management processes in the Kawasaki shipyards, and in the process became one of the fathers of modern management.

It was first used in the $1920 \mathrm{~s}$, and considered one of the $7^{\text {th }}$ basic tools of the quality control. Because of its shape It is known as a fishbone diagram, it similar to the shape of a fish skeleton.

It can be helpful tools for brainstorming potential change ideas or interventions help to solve the healthcare most common problems.

Stream mapping was used for analyze process of the workflow for inpatient and to divide it into its component parts. Eliminate and variation was minimized unnecessary steps in the remaining process.

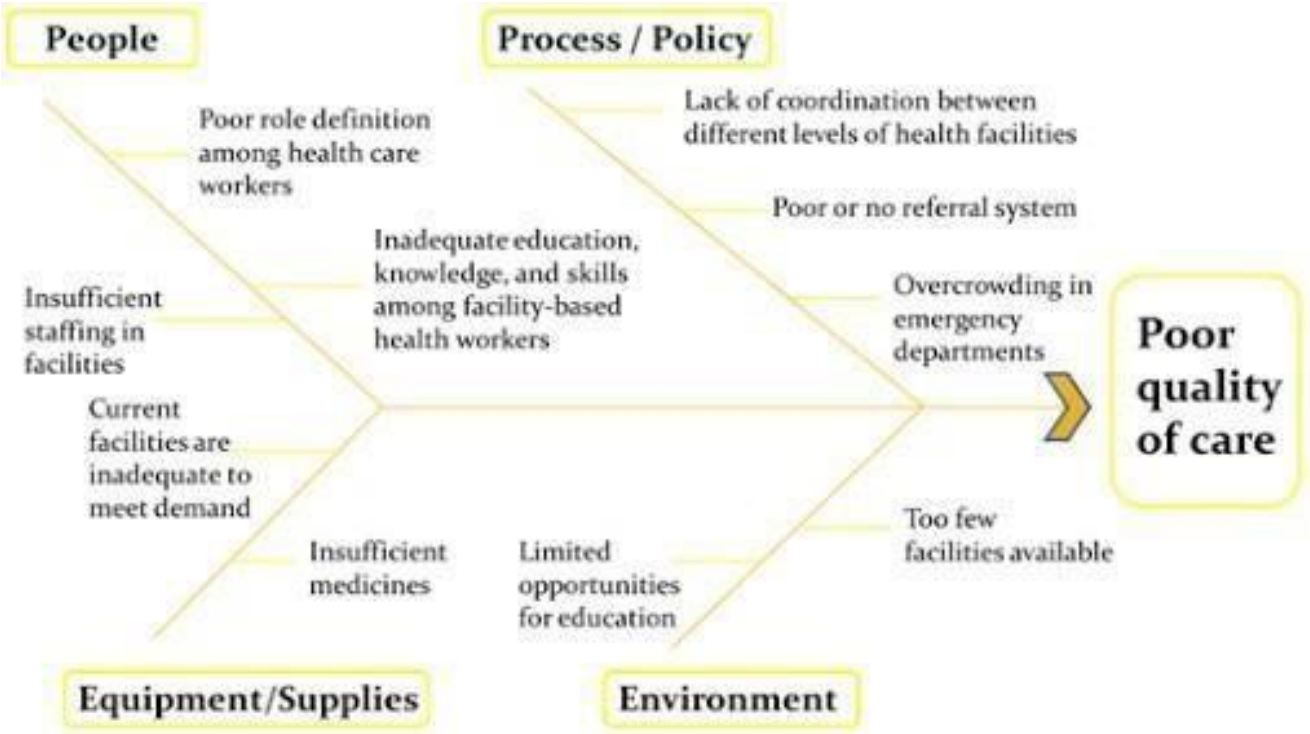




\section{$\underline{\text { Results }}$}

16 Risk Management practices to reduce malpractice \& improve

The Health Resources and Services Administration (HRSA), developed 16 practices for risk management to reduce medical errors and ensure patient safety on the basis of documentation review $\&$ interview with various stakeholders.

1- Program OF Quality improvement: Organization should take efforts to the quality of care improvement. These efforts may be review of clinical results data and applying long-term processes for enhancing the healthcare.

2- Clinical using of quality protocols: Organization must establish guidelines step by step for Appling effective clinical care to patients that the health care staff can use. These guidelines may be by professional organizations or even by the health Centre's staff.

3-Good communication with patients: Patients must be given all the information about their healthcare and treatment. Patients have a right to determine their received care and received all information about their health status in a way that they can understand. For example, providing the translation services as needed.

4- Good communication with providers: providers of Health care must receive good communication from their managers about their role and responsibility and they should communicate in a clear way with each other.

5- Patient Comprehensive medical records: We must mentioned All the care given to patients in an healthcare settings in detailed in patients' medical record. Patient record must be well maintained even paper or electronic.

6-the health care worker Credentialing: This is a process when all healthcare workers meet all the important educational and licensing requirements needed. The health Centre may conduct this activity. 
7- Informed consent Documentation: Patients must be informed about their care and treatment risks and benefits in form of informed consent.

8-Formal patient and staff grievances system: it is a system to collect, analyze, and focus on all the complaints received from patients or staff.

9- System of Incident reporting: It is a system to analyze and collect information on adverse events that occur in the health setting that result from errors or malpractice of care .

10- Peer review for patient: It is the check of medical records and patient health status by professionals in healthcare, within the health Centre or outside consultation, to provide the suitable care for patients.

11- Developing a process for assessing the risks of the health Centre and the actions to reduce its risks, this assessment can be made by the health Centre or outside consultants.

12- Tracking system for patient: It is an authorized system (electronic or paper) to ensure that important patients information such as tests results, missed appointments and any care received at different healthcare settings are evaluated and recorded.

13- Privileging process for healthcare practitioners: It is a process of verifying that health care practitioners have the convenient clinical competencies and the ability to perform effective clinical procedures.

14- Patient satisfaction surveys: It is a survey to evaluate patient satisfaction with the service level and health care level they received.

15- Staff training on risks management: Staff should receive inside and outside training on spots related to management of risks. 
16-Update policies and procedures for risks management: we should update written forms that explain the health setting's operations and processes for agreeing with management of risks.

\section{Discussion}

According to the results of the current study 1, Improve patient outcomes, staff interpretation, and professional development that results from a combined approach in how change is delivered, The delivery of health care with improved outcomes and costs reducing by continuous improving of work systems, Using a system change techniques and plans to improve patient healthcare experience and outcomes, To make a difference to patients by improving safety, effectiveness, and experience of care by helping them to understand of our complicated healthcare environment and applying changes using real time measurements for improvement.(BMJ ,2020).

According to the results of the current study 2, The clinical protocols allows to offer the suitable diagnostic treatment and healthcare services to patients, and quality training for clinical staff. By establishing protocols in patients' records it helps to save patient rights, the simplification of shared care. It may support the medico-legal strength of the delivered health care. If the protocols are detailed sufficiently, costing, coding and use of others resources information can flow directly from the clinical records. Such benefits may be increased by using protocols within the electronic patient record system. (ISSN: 0952-6862, 1994 ).

According to the results of the current study 3, by Using clear communication strategies with patients can help them to understand well any health information given to them oral. Also Communicating clearly helps patients to feel involved in their treatment, health progress and increases their desire to share in their treatment plans (Rockville, MD 20857, 2015).

According to the results of the current study 4, the communication between healthcare Staff is important for effective and timely processes. Today 
healthcare environment, communication technologies are important to connect healthcare professionals with other healthcare facilities, it is important also for ensuring best, effective, immediate care to patients.(OnPage, 14 august 2020).

According to the results of the current study 5, "The complete Health Record paper or electronic " contains all information about the patient's physical or mental status, that are recorded in any form and that are collected for the evaluation and treatment, including all records that prepared by a healthcare provider or other providers. (By Prabhat Dhar, 2018).

According to the results of the current study 6, Healthcare credentialing is important for many parties. Your doctor and other healthcare workers all need to have the education, training, and skills needed for proper care for patients. Healthcare organizations monitor the work of medical staff to evaluate the reports of malpractice of care. All of this reporting and monitoring must be checked continually by healthcare settings that employ the workers and by healthcare insurance companies want to approve workers lists, this details essential for credentialing for providers and offers recommendations on the responsibilities efficiently and effectively as possible as.(Becky Simon, 2019 ).

According to the results of the current study 7, The Joint Commission international need documentations of all the components of informed consent "in a form, progress notes in the record." The following are components for documentation of the informed consent, the procedures nature, risks and benefits of the procedures and the available alternatives, risks and benefits of it, and evaluate of the patient's understanding of these components, It is the involvement of the hospital to make it sure that the patient is participating in the decision-making and avoid to make the patients feel forced to agree about this. (StatPearls Publishing, 2021 ). 
According to the results of the current study 8, The grievance encourage the effective communication between patients and hospital staff in problems, or complaints . Patients must help to assist in formulating and submitting grievances for resolution or prevent of problems.

Patients must know that they have the right to have their concerns or complaints heard by upper levels of management. (Reliasmedia, 2000).

According to the results of the current study 9, we should accept the reports of incidents, near misses and injuries or errors we made, because they enable us to take action handle and reduce the chance of future injuries, illness or incidents (worksafe Tasmania, 2020).

According to the results of the current study 10, The peer review in clinical settings, is the process which the health care professionals, include nurses and pharmacists are evaluate each other's clinical performance.it is a specific process may be (such as physician peer review, nursing peer review). Today, the clinical peer review is most popular in hospitals, may also occur in surgical centers and large group practices. The purpose of peer review is to quality improvement and safety of care. It also serves to reduce the organization's malpractice and meet regular requirements of the organization. Peer review also supports healthcare organizations to assure that physicians are competent and practice professionally to meet the health needs of patients. (Wikipedia , 2021 )

According to the results of the current study 11, Healthcare Risk management included the clinical and management systems, reports and processes employed to reveal, monitor, evaluate, and risks prevention. Employing risk management, healthcare organizations systematically and proactively protect patient safety and the organization's assets, accreditation and help in community progress. (NEJM Catalyst, April 25 2018).

According to the results of the current study 12, the patient tracking system help a healthcare provider to access and monitor the progress of a patient 
condition through applying of care during their stay in hospital . that systems are part of the information system of patient and may affected by the person's electronic health record, where all information about patient is stored in, such as the system used by radiology departments to track patients also help the system to store medical images, the patient laboratory information system and patient discharge systems.(Wikipedia, 2020 ).

According to the results of the current study 13, Medical staff privileging is a process Focus on a certain scope and content of patient care services that authorized for a health care worker by a health care institution, based on the evaluation of the staff's credentials and performances. Joint Commission International Accreditation standards for Hospitals Privilege committee members who are involved in granting, denying, or terminating medical staff privileges have an ethical responsibility to be guided by professional practice \& regulations of the country which prevails ( Thomas Mathew ,2020).

According to the results of the current study 14, surveys for Patient satisfaction important for patient self-assessments of medical care they received. Depending on type of patient satisfaction is being measured; examples may include behaviors of staff, clinician way of communication, the technical skills and the hospital environment. As the patients are "satisfied" it depends on their expectations about the different touch points. (NEJM Catalyst ,January 1, 2018).

According to the results of the current study 15, the healthcare facilities and hospitals are increasing its programs for risks management that work on promote and enhance patient safety and prevent legal exposure, to ones that are increasingly display and view risks through the much wider lens of all the healthcare internal system . (NEJM Catalyst, April 25, 2018).

According to the results of the current study 16, update policies and procedures lead to reduce practice variability that may affect the care apply and cause patient harm. (AnneV. Irving, MA, FACHE, DFASHRM, 2014). 


\section{Conclusion}

Enhancement of patient care has become the first interest of all health care practitioners, to achieve the higher degree of patient satisfaction. Develop the awareness among the people, increase desire for better care, compete to be more keen, to be more health care regulating, As the medical malpractice and poor outcomes increased, we concern about this factors that lead to this change.

From this project we recommend the following:

1. Improve clinical care coordination.

2. Discharge efficiency

3. Standardize clinical communication

4. Making a process in which the entire institution can follow will make a space for questions and save our time .

5. Develop an interdisciplinary plan of care.

\section{Recommendations:}

From the result of this project the following are recommend:

1- Quality tools implementation should improve clinical care coordination.

2-Quality tools implementation should enhance coordination between different levels of health facilities.

3-Implementation of quality tools should standardize clinical communication.

4- Making a process in which all of the institution can follow will leave space for questions and save time and Develop an effective and complete plan of care.

5- Quality tools implementation should develop a competent plan of care. 6-Quality tools implementation should provide sufficient medicine to meet inpatient demands. 
7-Implementation of quality tools should provide adequate opportunities for improvement knowledge and experience of health care workers.

\section{Summery}

At the end of the research we find that the health institution one of the institutions that mostly use and depend on quality tools by improving the quality service to it patients.

The quality control has become one of most important requirements recent years the quality tools have a great rule in the improving the efficiency and effectiveness.

Our main cause for using quality tools is to improving patient care, reduce length of stay in hospitals and also reduce risks and errors that can be harmful to the patient.

When we use the quality tools in healthcare as fishbone we find that it help to maintain quality improvement, suitable use of quality protocols, good communications with providers, complete and integrate patient medical records, credential of healthcare staff, documentations of medical consents, formal patient complaint mechanism, internal system for report any incident, using of peer review way for patient cases, it also uses as a process to assess any risks occur in health Centre ,patient tracing system and regular patient satisfaction surveys.

At the end we recommend to: improve clinical care coordination, stream line discharge efficiency, standardize clinical communication, making a process in which all of the institution can follow, leave space for questions and save time and Develop a comprehensive plan of care.

\section{References}

- https://www.sciencedirect.com/science/article/abs/pii/S014600051730015 0\#f0010 
- https://en.m.wikipedia.org/wiki/Ishikawa_diagram

- http://www.qualityinhealthcare.com/2020/06/22/cause-and-effectdiagram-fishbone-diagram-or-ishikawa-diagram/ https://www.researchgate.net/publication/342732546_Application_of_Qua lity_Tools_and_Techniques_in_Hospital_Case_Study_in_Bandung_Indon esia

- https://www.tandfonline.com/doi/abs/10.1080/00185868.2017.1279514?jo urnalCode $=$ vhos $20 \&$

- https://www.researchgate.net/publication/342732546_Application_of_Qua lity_Tools_and_Techniques_in_Hospital_Case_Study_in_Bandung_Indon esia

- https://www.bmj.com/content/368/bmj.m865

- https://www.emerald.com/insight/content/doi/10.1108/0952686941007470 2/full/html

- https://www.ahrq.gov/health-literacy/improve/precautions/tool4.html

- https://www.onpage.com/effective-communication-between-healthcareprofessionals-best-practices/

- https://www.elationhealth.com/blog/chr/

- https://www.smartsheet.com/medical-provider-credentialing-guide

- https://www.reliasmedia.com/articles/45014-how-to-manage-patientgrievances

- https://www.ncbi.nlm.nih.gov/books/NBK430827/

- https://worksafe.tas.gov.au/topics/Health-and-Safety/managingsafety/getting-your-safety-systems-right/your-internal-incident-reportingsystem

- https://en.m.wikipedia.org/wiki/Clinical_peer_review

- https://catalyst.nejm.org/doi/full/10.1056/CAT.18.0197

- https://en.m.wikipedia.org/wiki/Patient_tracking_system 
- https://www.linkedin.com/pulse/medical-staff-privilege-gettingcomplicated-thomas-mathew

- https://catalyst.nejm.org/doi/full/10.1056/CAT.18.0197

- https://catalyst.nejm.org/doi/full/10.1056/CAT.18.0288

- https://www.psqh.com/analysis/policies-and-procedures-for-healthcareorganizations-a-risk-management-perspective/ 